\title{
Modelo lógico do "Programa Cidade Ativa, Cidade Saudável” de São José dos Pinhais, Paraná
}

Logic Model of “Cidade Ativa, Cidade Saudável Program” in São José dos Pinhais, Brazil.

\section{AUTORES \\ Alice Tatiane da Silva ${ }^{1,2}$ (D) \\ Letícia Pechnicki dos Santos ${ }^{1,3}$ (D) \\ Ciro Romelio Rodriguez-Añez ${ }^{1,3}$ (i) \\ Rogério César Fermino ${ }^{1,3,4}$ (D) \\ 1 Universidade Tecnológica Federal do Paraná Grupo de Pesquisa em Ambiente, Atividade Física e Saúde, Curitiba, Paraná, Brasil. \\ 2 Superintendência Geral do Esporte, Governo do Estado do Paraná, Curitiba, Paraná, Brasil. \\ 3 Universidade Tecnológica Federal do Paraná, Programa de Pós-Graduação em Educacão Física. Grupo de Pesquisa em Ambiente, Atividade Física e Saúde, Curitiba, Paraná, Brasil. \\ 4 Universidade Federal do Paraná, Programa de Pós-Graduacão em Educação Física, Curitiba, Paraná, Brasil. \\ CONTATO \\ Alice Tatiane da Silva silva.alice@outlook.com \\ Universidade Tecnológica Federal do Paraná \\ - Sede Neoville. Rua Pedro Gusso, 2.601, \\ Curitiba, Paraná, Brasil. \\ CEP: 81310-900. \\ DOI}

$10.12820 /$ rbafs. $26 \mathrm{e} 0193$

\begin{abstract}
RESUMO
O objetivo deste estudo foi desenvolver o modelo lógico do "Programa Cidade Ativa, Cidade Saudável" da cidade de São José dos Pinhais, Paraná. Trinta e seis gestores forneceram parte das informações e o modelo lógico foi elaborado conforme as diretrizes do Centers for Disease Control and Prevention. Foram identificadas 163 pessoas responsáveis por elaborar, gerenciar, coordenar, apoiar e ministrar diversas ações de atividades físicas, esportivas e lúdicas para a população, sob responsabilidade das divisões de "lazer e eventos populares", "esportes populares" e "esportes de rendimento". O modelo lógico possibilitou a identificação ordenada dos recursos, atividades, produtos e metas do programa. Estes dados poderão auxiliar a reestruturação do Programa frente a possíveis demandas de gestão. Futuros estudos podem utilizar estas informações para avaliar a efetividade e a eficácia do Programa nos indicadores de saúde da população.
\end{abstract}

Palavras-chave: Atividade motora; Atividades de lazer; Promoção da saúde; Avaliação de programas e projetos de saúde; Política pública.

\section{ABSTRACT}

This study aimed to develop the logical model of "Programa Cidade Ativa, Cidade Saudável" in São José dos Pinhais, Brazil. Thirty-six managers provided part of information and the logical model was developed according to guidelines of Centers for Disease Control and Prevention. Were identified 163 people responsible for elaborating, managing, coordinating, supporting and ministering actions of physical activities, sports and leisure activities for population, by responsibility the divisions of "leisure and popular events", "popular sport" and "sport performance". The logical model enabled the orderly identification the program's resources, activities, products and objectives. These data may help to restructure the program by possible demands of the management. Future studies can use this information to evaluate the effectiveness and efficiency of program in population's health indicators.

Keywords: Motor activity, Leisure activities; Health promotion; Evaluation of health programs and projects; Public policy.

\section{(cc) BY}

Este trabalho está licenciado com uma Licença

Creative Commons - Atribuição 4.0 Internacional.

\section{Introdução}

Devido ao fato de a inatividade física ser a quarta causa de mortalidade prematura a nível global ${ }^{1,2}$, diversos programas de promoção da atividade física a nível comunitário têm sido desenvolvidos em todo o mundo ${ }^{3,4}$. Recentemente, a World Health Organization ${ }^{5}$ estabeleceu estratégias globais para o aumento da atividade física, em que estes programas assumem um importante papel.

$\mathrm{Na}$ América Latina, as experiências de alguns programas foram documentadas na literatura ${ }^{3,6-9}$. No Brasil, a Política Nacional de Promoção da Saúde guia as ações e a inserção de programas para a promoção da atividade física na comunidade ${ }^{10}$, sendo esta conside- rada uma estratégia promissora, a qual pode contribuir para a prevenção de doenças crônicas, o desenvolvimento do capital social, aumento da qualidade de vida, entre outros cobenefícios ${ }^{3,4,10}$.

Em São José dos Pinhais, Paraná o "Programa Cidade Ativa, Cidade Saudável" foi implementado no ano de 2017 pela Secretaria Municipal de Esporte e Lazer (SEMEL), projetado como uma ação multissetorial, em parceria com as Secretarias de Saúde, Educação e Assistência Social, para melhorar os indicadores de saúde da população e incentivar a prática de atividades físicas e hábitos alimentares saudáveis da população de maneira orientada e permanente. 
O desenvolvimento do modelo lógico de programas de promoção de atividades físicas possibilita a sistematização e o esclarecimento das estratégias, recursos necessários e os objetivos destas intervenções ${ }^{11}$. Portanto, faz-se necessário o desenvolvimento do modelo lógico do "Programa Cidade Ativa, Cidade Saudável", para que seja possível identificar e descrever de maneira estruturada os recursos, atividades, produtos e metas para o seu desenvolvimento. Também, este procedimento é importante para possibilitar a avaliação da efetividade e eficácia das ações nos indicadores de saúde da população, bem como para a avaliação da sua viabilidade econômica ${ }^{3,8,11}$. Ainda, de maneira complementar, a sistematização destas informações pode servir como "modelo de conduta" para as ações de Secretarias de Esporte e Lazer de outras cidades do Brasil.

Portanto, o objetivo deste estudo foi desenvolver o modelo lógico do "Programa Cidade Ativa, Cidade Saudável” de São José dos Pinhais, Paraná.

\section{Métodos}

O estudo foi aprovado na Comissão Nacional de Ética em Pesquisa (CONEP) com o Certificado de Apresentação de Apreciação Ética (CAAE) sob o número de protocolo 88214618.0.0000.5547 e parecer do Comitê de Ética em Pesquisa (CEP) sob o número 2.759.583, da Universidade Tecnológica Federal do Paraná. Os participantes foram consultados, esclarecidos sobre a voluntariedade e aceitaram participar da pesquisa por meio da assinatura do Termo de Consentimento Livre e Esclarecido (TCLE), de acordo com as recomendações do Sistema Nacional de Ética em Pesquisa do Conselho Nacional de Saúde.

A pesquisa foi conduzida entre os meses de junho e agosto de 2018. Participaram deste estudo todos os 36 gestores da administração pública da cidade (quatro secretários, três chefes de divisão, 29 coordenadores), intencionalmente selecionados por estarem diretamente ligados ao desenvolvimento das ações e atividades do Programa.

Os dados foram coletados com métodos mistos de pesquisa. As informações qualitativas foram coletadas com questionário estruturado auto aplicado, enquanto os dados quantitativos foram coletados por meio de planilhas e relatórios de gestão do período de agosto de 2017 a agosto de 2018.

Para a elaboração da primeira versão do modelo lógico, foi desenvolvido um questionário para coletar as informações sobre recursos, ações, atividades e ob- jetivos do Programa. A coleta das informações ocorreu via e-mail, com aplicação de formulário eletrônico, em que participaram desta etapa apenas os secretários da administração pública $(n=4)$.

Após a elaboração da primeira versão do modelo lógico, os 36 gestores participaram de uma reunião de consenso em que foram apresentados os objetivos, a estrutura e os resultados da versão preliminar do modelo lógico. Durante quatro horas de discussão, todos revisaram a estrutura e o conteúdo do modelo, validaram as informações colaboraram com dados adicionais para que fosse possível elaborar a versão final do documento.

O modelo lógico foi desenvolvido de acordo com as diretrizes propostas pelo Centers of Disease Control ${ }^{11}$, que prevê a avaliação de programas de promoção da atividade física com a descrição sistemática dos recursos, atividades, produtos e metas a curto, médio e longo prazo. Ainda, a versão final do modelo lógico foi estruturada com base em documentos similares de outros programas de atividade física a nível comunitário desenvolvido em cidades da América Latina ${ }^{6-9}$.

\section{Resultados}

O "Programa Cidade Ativa, Cidade Saudável” foi implementado no ano de 2017, como iniciativa da SEMEL e conta com a colaboração de instituições governamentais (municipal e federal), comunitárias e da iniciativa privada (Figura 1). No entanto, é importante ressaltar que diversas atividades eram desenvolvidas pela SEMEL antes de 2017, mas a implementação do Programa possibilitou a reorganização sistemática destas ações, o que contribuiu para a otimização de recursos e ampliação do atendimento e oferta de diversas ações e atividades para a população.

\section{Recursos}

Fazem parte do quadro do Programa 163 funcionários alocados na SEMEL (Recursos humanos: 1 Secretário Municipal de Esporte e Lazer, 3 diretores, 8 agentes administrativos, 4 chefes de divisão, 28 coordenadores, 72 estagiários, 23 professores terceirizados, 3 professores voluntários, 1 estagiário de ensino médio, 3 motoristas, 9 funcionários de manutenção, 8 funcionários de serviços gerais), subdivididos em quatro divisões: 1) Lazer e Eventos Populares: $O$ chefe de divisão e os coordenadores são responsáveis por gerir e desenvolver os programas, atividades e eventos de lazer otimizando a utilização dos espaços públicos de lazer do município. Os professores horistas e estagiários são responsáveis 


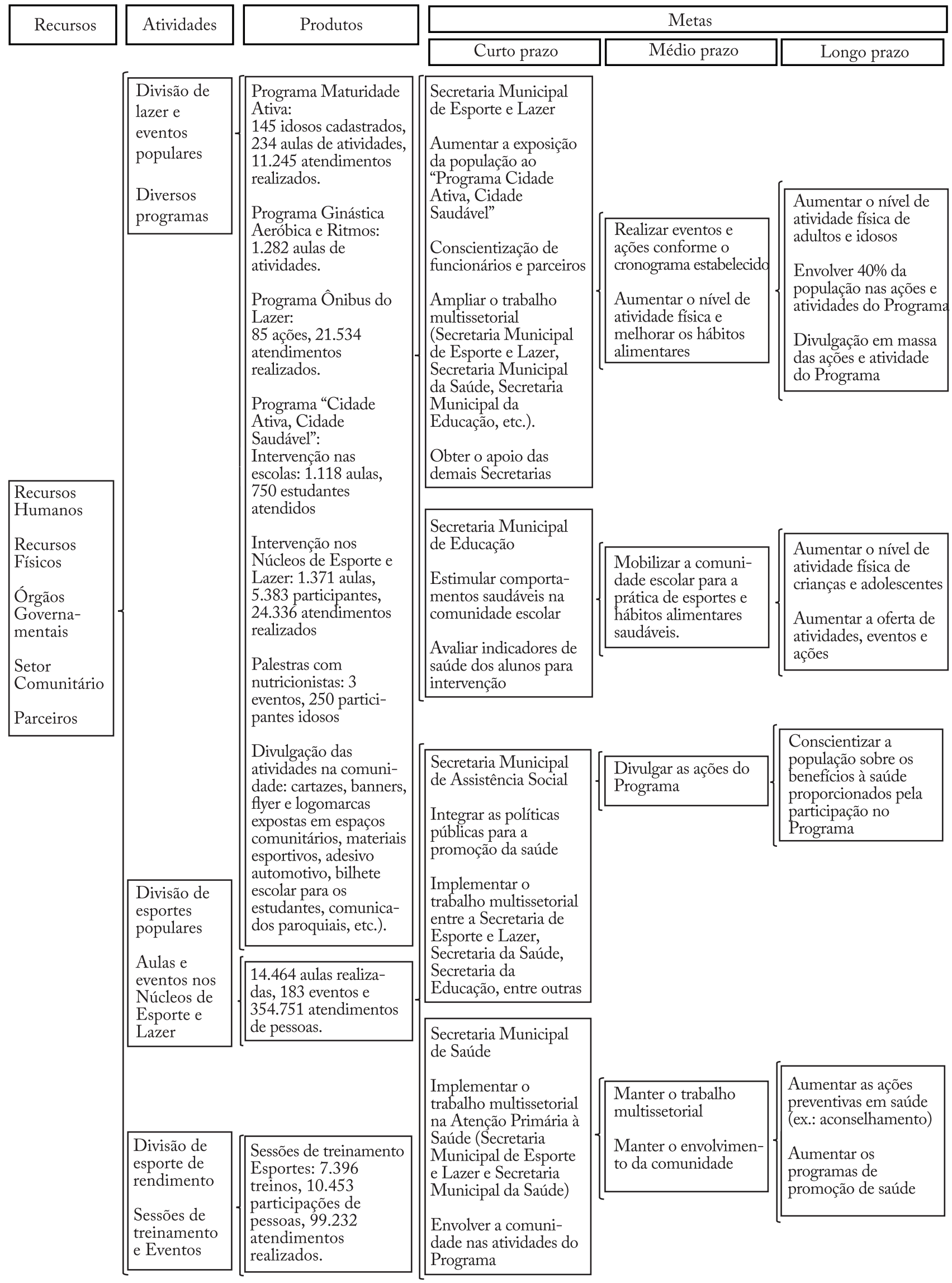

Figura 1 - Modelo lógico do "Programa Cidade Ativa, Cidade Saudável” de São José dos Pinhais, Paraná (2017-2018). 
por conduzir e aplicar as atividades do programa na comunidade; 2) Esportes Populares: O chefe de divisão e os coordenadores são responsáveis por gerenciar e desenvolver intervenções em atividades físicas e esporte para a comunidade nos Centros e Núcleos de Esporte e Lazer. Os professores horistas são responsáveis por ministrar aulas específicas, como as de lutas e dança. Os estagiários auxiliam os coordenadores nas atividades diárias dos Núcleos de Esporte e Lazer; 3) Esporte de Rendimento: $\mathrm{O}$ chefe de divisão e os coordenadores são responsáveis pela gestão e promoção de ações que desenvolvam o esporte de base, amador e profissional no município; 4) Atendimento Operacional: $\mathrm{O}$ chefe de divisão é responsável por coordenar as ações e os auxiliares de manutenção e de serviços gerais são responsáveis pela manutenção e zelo dos espaços públicos para a prática de atividades físicas da cidade. Vale ressaltar que, todas as divisões possuem atribuições no programa, para desenvolver as intervenções ou promover a divulgação do mesmo.

O programa conta com os seguintes recursos físicos: 2 ônibus do lazer, 15 Núcleos de Esporte e Lazer, Academias ao ar livre, Parques, Praças, Ruas, Pistas para caminhada, Bosques, Espaços livres, Quadras, Canchas, Campos, Pistas de skate, Campos de futebol, Centros de treinamento, Centro de excelência, Unidades Básicas de Saúde, Igrejas, Escolas, Sala de musculação, Centro Municipal de Ensino Infantil, Associação de Moradores.

Os recursos financeiros são provenientes, em sua maior parte, da SEMEL sendo aplicada toda a verba destinada à secretaria para o desenvolvimento do Programa no período (aproximadamente $\mathrm{R} \$ 8.000 .000,00$ ). Cerca de $63 \%$ do total $(\mathrm{R} \$ 5.000 .000,00)$ foram utilizados em pagamento de funcionários.

Os colaboradores do programa pertencem aos mais variados segmentos da comunidade, entre eles órgãos governamentais, iniciativas comunitárias e empresas do setor privado [(órgãos governamentais: Secretaria Municipal de Educação, Secretaria Municipal de Saúde, Secretaria Municipal de Assistência Social, Secretaria Municipal de Urbanismo, Secretaria Municipal de Agricultura, Secretaria Municipal Indústria, Comércio e Turismo, Secretaria Municipal da Cultura, Universidade Tecnológica Federal do Paraná, Guarda Municipal); (setor comunitário: Conselho Municipal do Esporte, Associação de Incentivo ao Esporte e Lazer, Associação de Funcionários Públicos Municipais); (parceiros: Conselho Regional de Educação Fí- sica, Serviço Social da Indústria, Serviço Nacional de Aprendizagem Comercial, Associação Empresarial de São José dos Pinhais, Ordem dos Advogados do Brasil, Criarte, Telex, Supermercados Muffato, Drogarias Paraná, Shopping São José, Colégio Tradição, Hospital Nova Clínica, Restaurante América Grill, Cooperflux Transportes Rodoviários, GAMMA ferramentas, Restaurante Parada de Minas, Instituo Menna Barreto)].

\section{Atividades}

A Divisão de Lazer e Eventos Populares oferta programas como 1) Maturidade Ativa (público-alvo: idosos; aulas de alongamento, dança, festival esportivo, jogos adaptados, equipes de rendimento esportivo, palestras, torneios esportivos, jogos esportivos, eventos temáticos); 2) Ginástica Aeróbica e Ritmos (público-alvo: jovens e adultos; aulas de ginástica aeróbica e ritmos, eventos temáticos; 3) Ônibus do Lazer (público-alvo: crianças e adolescentes; atividades de danças, jogos pré-desportivos, competitivos e cooperativos, camarim de pintura, jogos de tabuleiro, cama elástica, slack line, brincadeiras tracionais e de palco, teatro); 4) Eventos populares do "Programa Cidade Ativa, Cidade Saudável” (público-alvo: população em geral; intervenção nas escolas e Núcleos de Esporte e Lazer; palestras com nutricionistas; divulgação das atividades na comunidade; festivais com aulão de ginástica aeróbica e ritmos, corrida kids, gincana pais e filhos, atendimentos em saúde, feira de orgânicos; circuito de corrida de rua, premiação do ranking de corrida de rua, circuito de corrida kids, festa do dia da criança, dia do lazer acessível, aniversário da cidade).

Na Divisão de Esportes Populares, o esporte é desenvolvido no âmbito comunitário nos Núcleos de Esporte e Lazer, com a oferta de aulas e eventos de atividades de iniciação esportiva e esportes coletivos, lutas, aulas de ginásticas e ritmos, caminhada e corrida de rua, tênis de mesa, xadrez e atividades para a terceira idade [aulas: esportes coletivos (vôlei, futsal, basquete e handebol), iniciação esportiva (vôlei, futsal, basquete, handebol, atletismo), lutas (karatê, judô, jiu-jitsu, capoeira, artes marciais mistas, muaythay, taekwondo, luta olímpica), ginástica (ginástica aeróbica e ritmos, circuito funcional, jump, step, Pilates, cross-trainning), dança (ritmos e dança gaúcha), caminhada orientada, tênis de mesa, xadrez, atividades para terceira idade (alongamento, caminhada, jogos adaptados); eventos:

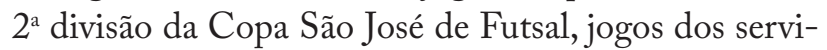
dores municipais (corrida de rua, natação, futsal, vôlei 
de quadra e de praia, truco, basquete, handebol, xadrez, tênis de quadra e de mesa, bocha), circuito inter Núcleos (vôlei, basquete, futsal handebol).

A Divisão de Esporte de Rendimento é responsável por desenvolver o esporte a nível competitivo, seja esporte de base, amador e rendimento. São desenvolvidas as modalidades de vôlei de quadra e praia, futebol, futsal, basquete, handebol, tênis de mesa, xadrez, atletismo e ginástica rítmica. A oferta destas atividades e a frequência com que são desenvolvidas varia de acordo com a divisão e o local de atendimento [sessões de treinamento: esportes (vôlei de quadra e praia, futebol, futsal, basquete, handebol, tênis de mesa, atletismo, ginástica rítmica, xadrez); eventos: campeonato amador de futebol de campo (adulto e juvenil), circuito escolar (xadrez e tênis de mesa), copa São José de futebol de campo (categoria todas as idades e categoria "veterano"), jogos escolares do Paraná "bom de bola”, jogos escolares do Paraná fases municipais e macrorregional, jogos Inter Núcleos de Esporte e Lazer, festivais internos de modalidades)].

\section{Produtos}

No período de agosto de 2017 a agosto de 2018, a Divisão de Lazer e eventos populares realizou cerca de 4.114 ações (entre elas aulas de atividades físicas e eventos de lazer) e efetuou 146.461 atendimentos de pessoas. A Divisão de Esportes Populares promoveu 14.647 aulas e eventos esportivos, totalizando 354.751 atendimentos. Por fim, a Divisão de Esportes de Rendimento ofertou 7.396 sessões de treinamento e eventos esportivos realizando 99.232 atendimentos.

Para a adequada interpretação dos produtos do programa, é importante ressaltar que os números não refletem necessariamente a quantidade de pessoas cadastradas nas ações e/ou atividades do programa, mas sim o número de atendimentos realizados. Por exemplo, 145 idosos estavam devidamente cadastrados no "Programa Maturidade Ativa", mas foram realizados 11.245 atendimentos. Esta diferença ocorre pela possibilidade do mesmo idoso, com cadastro atualizado, ser atendido (recebido orientação, realizado aulas, etc.) diversas vezes ao ano. Ou ainda, pela participação esporádica de idosos não cadastrados em "festivais" para a divulgação do programa.

\section{Metas}

Foram identificadas metas a curto, médio e longo prazo. A curto prazo, espera-se: 1) aumentar a exposição da população ao Programa (conhecimento e participação); 2) conscientizar os funcionários e os parceiros sobre a importância da ampliação do Programa; 3) ampliar o trabalho multissetorial (Secretaria Municipal de Esporte e Lazer, Secretaria Municipal da Saúde, Secretaria Municipal da Educação, etc.); 4) obter apoio das demais secretarias para o desenvolvimento do Programa; 5) estimular comportamentos saudáveis na comunidade escolar (estudantes, pais e professores); 6) avaliar indicadores de saúde na comunidade escolar; 7) integrar as políticas de promoção de saúde; 8) implementar o trabalho multissetorial entre as secretarias; 9) implementar o trabalho multissetorial na Atenção Primária à Saúde (Secretaria Municipal de Esporte e Lazer e Secretaria Municipal da Saúde); 10) Envolver a comunidade nas atividades do Programa.

A médio prazo espera-se 1) realizar as ações conforme o cronograma; 2) aumentar o nível de atividade física e melhorar os hábitos alimentares; 3) mobilizar a comunidade escolar para a prática de esportes e de hábitos alimentares saudáveis; 4) divulgar as ações do Programa; 5) manter o trabalho multissetorial; e 6) manter o envolvimento da comunidade.

A longo prazo pretende-se 1) aumentar o nível de atividade física de adultos e idosos; 2) envolver cerca de $40 \%$ da população nas ações e atividades do programa; 2) realizar a divulgação em massa das atividades e ações do Programa; 4) aumentar o nível de atividade física de crianças e adolescentes; 5) aumentar a oferta de atividades e ações; 6) conscientizar a população sobre os benefícios à saúde proporcionados pela participação no Programa; 7) aumentar as ações preventivas (ex.: aconselhamento em saúde); e 8) os programas de promoção da saúde na Atenção Primária à Saúde.

Por fim, o objetivo geral do "Programa Cidade Ativa, Cidade Saudável” é melhorar os indicadores de saúde da população, incentivando a prática de atividades físicas e hábitos alimentares saudáveis de maneira orientada e permanente. Como objetivos específicos espera-se: 1) incentivar as ações de promoção da atividade física e de hábitos alimentares saudáveis; 2) aumentar o conhecimento do Programa; 3) conscientizar a população sobre os benefícios à saúde alcançados pela participação no Programa; 4) avaliar a eficácia e a efetividade do Programa nos indicadores de saúde da população.

\section{Considerações finais}

O desenvolvimento do modelo lógico do "Programa Cidade Ativa, Cidade Saudável” permitiu identificar os 
recursos, atividades, produtos e metas propostas para as ações e atividades desenvolvidas. Verificou-se neste processo ampla atuação da administração pública, que cede ao Programa as principais estruturas (recursos humanos, recursos financeiros, insumos) necessários para a sua realização. Ainda, foram identificados parceiros em diversos segmentos, demonstrado uma boa aceitação do programa pela comunidade.

Estes dados poderão auxiliar a reestruturação do Programa frente a possíveis demandas de gestão. Futuros estudos podem utilizar estas informações para avaliar a efetividade e a eficácia do Programa nos indicadores de saúde da população.

\section{Conflito de interesse}

Os autores declaram não haver conflitos de interesse.

\section{Contribuição dos autores}

Silva AT, participou na concepção inicial deste estudo, coleta e análise dos dados, revisão da literatura e redação do manuscrito em todas as suas etapas. Santos LP, participou da redação e revisão crítica do manuscrito. Rodriguez-Añez CR, participou da redação e revisão crítica do manuscrito. Fermino RC, foi responsável pela concepção do manuscrito, coordenação científica do projeto de avaliação do programa, participou da redação do manuscrito em todas as suas etapas, além da revisão crítica do texto. Todos os autores aprovaram a versão final do manuscrito.

\section{Agradecimento}

Os autores agradecem os integrantes do Grupo de Pesquisa em Ambiente, Atividade Física e Saúde (GPAAFS/UTFPPR) pela colaboração na coleta dos dados. A Prefeitura e a Secretaria Municipal de Esporte e Lazer de São José dos Pinhais (SEMEL) pela colaboração e abertura do campo de pesquisa.

\section{Referências}

1. Ekeiund U, Steene-Johannessen J, Brown WJ, Fagerland W, Owen N, Powell KE, et al. Does physical activity attenuate, or even eliminate, the detrimental association of sitting time with mortality? A harmonised meta-analysis of data from more than 1 million men and women. Lancet. 2016;388(10051):1302-10.
2. Lee I-M, Shiroma EJ, Lobelo F, Puska P, Blair SN, Katzmarzyk PT. Effect of physical inactivity on major noncommunicable diseases worldwide: an analysis of burden of disease and life expectancy. Lancet. 2012;380(9838):219-29.

3. Heath GW, Parra DC, Sarmiento OL, Andersen LB, Owen N, Goenka S, et al. Evidence-based intervention in physical activity: Lessons from around the world. Lancet. 2012;380(9838):272-81.

4. Reis RS, Salvo D, Ogilvie D, Lambert EV, Goenka S, Brownson RC. Scaling up physical activity interventions worldwide: stepping up to larger and smarter approaches to get people moving. Lancet. 2016;388(10051):1337-48.

5. World Health Organization. Global action plan on physical activity 2018-2030: more active people for a healthier world. Geneva. 2018. [citado em 2018 mar 3]. Disponível em: https://www.who.int/ncds/prevention/physical-activity/ global-action-plan-2018-2030/en/.

6. Rios AP, Paez DC, Pinzón EA, Fermino RC, Sarmiento OL. Logic model of the Recreovía: a community program to promote physical activity in Bogota. Rev Bras Ativ Fís Saúde. 2017;22(2):206-12.

7. Ribeiro IC, Torres A, Parra DC, Reis RS, Hoehner C, Schmid TL, et al. Using logic models as iterative tools for planning and evaluating physical activity promotion programs in Curitiba, Brazil. J Phys Act Health. 2010;7(Suppl 2):S15562.

8. Silva AT, Hino AAF, Reis RS. Incentivo à ciclomobilidade em Curitiba-PR: desenvolvimento do modelo lógico dos programas Pedala Curitiba e Ciclolazer. Rev Bras Ativ Fís Saúde. 2017;22(5):493-97.

9. Vidigal JMS, Caldeira JRS, Fermino RC, Martins-Costa HC, Fernandes AP. Ação "Lazer Mais Saúde": experiências em promoção da atividade física nas Academias a Céu Aberto em Belo Horizonte, Minas Gerais, Brasil. Rev Bras Ativ Fís Saúde. 2019;24:e0105.

10. Brasil. Ministério da Saúde. Secretaria de Vigilância em Saúde. Política Nacional de Promoção da Saúde. Ministério da Saúde, Secretaria de Atenção à Saúde - Brasília: Ministério da Saúde. 2018. [citado em 2020 mai 10]. Disponível em: http://bvsms.saude.gov.br/bvs/publicacoes/ politica_nacional_promocao_saude.pdf.

11. United States Departament of Health and Human Services. Centers for Disease Control and Prevention. Physical Activity Evaluation Handbook. Atlanta, Georgia, GA. United States of America. 2002. [citado em 2017 ago 3]. Disponível em: https://www.cdc.gov/nccdphp/dnpa/physical/health_ professionals/interventions/handbook_508.pdf.

Recebido: 24/07/2020

Aprovado 24/09/2020 